and did not unravel any new features of the staurolite structure. Instead, the X-ray structure was confirmed. In the future, procedures can be refined and become more routine as the method is applied to other examples with known structure to test the reliability and to unknowns.

HRW remembers a $\mathrm{PhD}$ qualifying examination in the Department of Biophysics and following discussions with Professor R. Glaeser which initiated this interdisciplinary research. J. Arnoth supplied the specimen of staurolite and A. C. Larson kindly helped with the stereoplot. Comments from two reviewers improved the manuscript. Financial support from NSF Grants EAR-88 16577 and 9104605 (to HRW), NIH Grant GM-36884 (to KHD) and from the UC Education Abroad Program (to HM and HRW) is gratefully acknowledged. KHD is supported by the Assistant Secretary of the Office of Environmental and Health Research, Office of Energy Research, MAO'K and NCEM are supported by the Director, Office of Basic Energy Sciences, Materials Science Division, all through the US Department of Energy under Contract No. DE AC-03-76SF0098. HRW and $\mathrm{HM}$ are grateful for generous access to facilities at NCEM and for assistance by $\mathrm{C}$. Nelson and D. Ah Tye. HRW is appreciative of the hospitality at CMS LANL on the occasion of a leave.

\section{References}

Amos, L. A., Henderson, R. \& Unwin, P. N. T. (1982). Prog. Biophys. Mol. Biol. 39, 183-231.

Brillinger, D. R., Downing, K. H. \& Glasser, R. M. (1990). J. Stat. Plan. Inference, 25, 235-259.

De Rosier, D. J. \& KLUG, A. (1968). Nature (London), 217, 130-134.

De Rosier, D. J. \& Moore, P. B. (1970). J. Mol. Biol. 52, 355. Downing, K. H., Hu, M., Wenk, H.-R. \& O'KeEFE, M. A. (1990). Nature (London), 348, 525-528.
Epicier, T., O’Keefe, M. A. \& Thomas, G. (1990). Acta Cryst. A46, 948-962.

Frank, J. (1973). Optik (Stuttgart), 38, 519-536.

Glaeser, R. M. (1985). Ann. Rev. Phys. Chem. 36, 243-275.

Gronsky, R. (1980) 38th Ann. Proc. Electron Microsc. Soc. Am. San Francisco Press.

HAINISCH, K. (1966). N. Jb. Mineral. Monatsh. pp. 362-366.

Henderson, R., Baldwin, J. M., Ceska, T., Zemlin, F., Beckmann, E. \& Downing, K. H. (1990). J. Mol. Biol. 213, 899-929.

Henderson, R. \& UNwiN, P. N. T. (1975). Nature (London), 257, 28-32.

Hetherington, C. J. D., Nelson, E. C., Westmacott, K. H., Gronsky, R. \& Thomas, G. (1989). Mater. Res. Soc. Symp. Proc. 139, 277-282.

Hovmöller, S., Sjögren, A., Farrants, G., SundberG, M. \& MARINDER, B. O. (1984). Nature (London), 311, 238-241.

International Tables for Crystallography (1989). Vol. A. Dordrecht: Kluwer Academic Publishers.

International Tables for X-ray Crystallography (1974). Vol. IV, Table 2.4.6A, p. 155. Birmingham: Kynoch Press. (Present distributor Kluwer Academic Publishers, Dordrecht.)

JaCOB, J. (1941). Schweiz. Mineral. Petrogr. Mitt. 21, 124.

Juurinen, A. (1956). Ann. Acad. Sci. Fenn. Ser. A 3, 47, 1-53.

KilaAs, R. (1987). 45th Ann. Proc. Electron Microsc. Soc. Am., Baltimore, pp. 66-69. San Francisco Press.

Meike, A., Wenk, H.-R., O'Keefe, M. A. \& Gronsky, R. (1988). Phys. Chem. Miner. 15, 427-437.

NÁRAY-SZABó, I. (1929). Z. Kristallogr. 71, 103-116.

NÁray-SzaBó, I. \& SASVÁRI, K. (1958). Acta Cryst 11, 862-865.

O'Keefe, M. A. (1979). 37th Ann. Proc. Electron Microsc. Soc. Am., San Antonio, TX, pp. 556-557. Baton Rouge: Claitor.

O'Keefe, M. A., Buseck, P. R. \& IIJimA, S. (1978). Nature (London), 274, 322-324.

RAMACHANDRAN, G. N. \& SRINIVASAN, R. (1970). Fourier Methods in Crystallography. New York: Wiley.

Saxton, W. O. (1986). 44th Ann. Proc. Electron Microsc. Soc. Am. San Francisco, pp. 526-529. San Francisco Press.

Smith, D. J., Camps, R. A., Freeman, L. A., O'Keefe, M. A., SAXTON, W. O. \& WOOD, G. J. (1985). Ultramicroscopy, 18, 63-76.

SMith, J. V. (1968). Am. Mineral. 53, 1139-1155.

Stout, G. H. \& JENSEN, L. H. (1989). X-ray Structure Determination. A Practical Guide. New York: Wiley.

TAKÉUChI, Y. \& AIKaWA, N. (1972). Z. Kristallogr. 136, 1-22.

Veblen, D. R. \& BuSECK, P. R. (1979). Science, 206, 1398-1400.

WENK, H.-R. (1980). Am. Mineral. 65, 766-769.

Zvyagin, B. B. (1967). Electron Diffraction Analysis of Clay Mineral Structures. New York: Plenum.

Acta Cryst. (1992). A48, 716-727

\title{
The Domain Matrix Method: a New Calculation Scheme for Diffraction Profiles
}

\author{
By S. Pflanz and W. Moritz \\ Institut für Kristallographie und Mineralogie der Universität, Theresienstrasse 41, \\ D-8000 München 2, Germany
}

(Received 25 October 1991; accepted 10 March 1992)

\begin{abstract}
A new calculation scheme for diffraction profiles is presented that combines the matrix method with

$0108-7673 / 92 / 050716-12 \$ 06.00$
\end{abstract}

domain approaches. Based on a generalized Markov chain, the method allows the exact solution of the diffraction problem from any one-dimensionally disordered domain structure. The main advantage of this

(C) 1992 International Union of Crystallography 
model is that a domain statistic is used instead of a cell statistic and that the domain-length distribution can be chosen independently from the domain-type stacking. A recursive relation is derived for the correlations between the domains and a double recursive algorithm, not reducible to a simpler one, is obtained as solution. The algorithm developed here is referred to as the domain matrix method. Results and applications of the new approach are discussed.

\section{Introduction}

Until now, the calculation of reflection profiles for one-dimensionally disordered structures has been restricted to two main approaches. These are, firstly, the matrix method and, secondly, a numerical calculation of the Patterson function in a domain model. Both methods have their specific advantages and applications. The matrix or Markov-chain method is well suited for systems that can be described with one or a few short-range order parameters. Its main advantage is the analytic solution showing the relationship of peak positions and widths with the statistical parameters of the model. The matrix method, however, becomes cumbersome for domain distributions that correspond to higher-order Markov chains or non-Markov chains.

The numerical calculation of the correlation function and the reflection profile, on the other hand, has the advantage that more general distributions can be handled, but the relation between the statistical parameters of the system and the reflection profile is not analytically given and tedious model calculations are required. Solutions published until now have been only for special cases.

In this paper we derive a general calculation scheme that is practically applicable to all kinds of domain distributions and includes domains differing in structure and lattice orientation. The main advantage of the method is that the lengths of the domains can be chosen independently from their stacking. The calculation combines the matrix method with the numerical calculation of the Patterson function by introducing domain sequence probabilities and an iteration scheme for the numerical calculation of the sequence probabilities.

The first solutions of the diffraction problem for one-dimensionally disordered structures go back to Landau (1937) and Lifschitz (1937), Wilson (1942) and Hendricks \& Teller (1942). A general profilecalculation scheme based on these sources was developed by Jagodzinski (1949) and later by Kakinoki \& Komura $(1952,1954,1965)$, who, in conjunction with Jagodzinski, introduced the name 'matrix method' (MM). Since then, the MM has been studied quite extensively. Applications to crystallographic problems have been given by many authors in the last decades. For a recent overview see, for example, Jagodzinski (1987) and Jagodzinski \& Frey (1992). The conceptual foundation of the MM has also been established by Welberry (1985), who studied very general disorder problems using that approach. More recently, Seul \& Torney (1989) extended the matrix method to systems with nonstationary transition probabilities. This approach is related to our method since it extends the near-neighbour correlations to more general domain distributions yet is restricted to the special case of a layer stacking with only two different spacings.

Besides bulk distribution problems, the disordering of surfaces has also been studied by the MM. Jagodzinski, Moritz \& Wolf (1978) extended the method to include multiple scattering effects for lowenergy electron diffraction from disordered or rough surfaces. Disorder models for steps at surfaces (stepterrace structures) have been developed by $\mathrm{Lu} \mathrm{\&}$ Lagally (1982), Pimbley \& Lu (1984, 1985a,b), Presicci \& Lu (1984), Pimbley, Lu \& Wang (1985), Fenter \& Lu (1985) and Lent \& Cohen (1984). The most general work, is that of Pukite, Lent \& Cohen (1985), who described the profiles for stepped surfaces with a varying terrace distribution.

Some of these examples already show that, besides simple Markov processes, more complicated nonMarkov processes may enter in the general description of disorder. This is in general the case for nonequilibrium structures, lamellar exsolution processes and (imperfectly) modulated structures. For such cases, the calculation of the intensity distribution in reciprocal space for a given distribution function of defects has been described by Hosemann \& Bagchi (1962) (paracrystal model). Houston \& Park (1970, 1971) extended this method to describe antiphase domains in adsorbate layers on surfaces. They used a numerical method and an approximation in the averaging process. A direct calculation of the Patterson function for the case of random stacking faults has been given by Cowley (1976) and Cowley \& Au (1978), which in this case leads to an analytic formula for the reflection profile. Recently, Neder, Frey \& Schulz (1990) developed a calculation for threedimensional disordered structures using a quite general model of disorder (microdomain model). The most general approach, until now, to one-dimensionally disordered domain structures is due to Adlhart (1981), who used a cyclic sequence of domains without limitations for the domain distributions. It is the main goal of this paper to deal with such nonMarkov models for one-dimensional disorder and to calculate the intensity distribution in reciprocal space by a quite general scheme.

The organization of the paper is as follows. In $\S 1$ we briefly describe the MM by using cell statistics. In $\S 2$ we develop the domain matrix method (DMM) by going from cell to domain statistics. In $\S 3$ we discuss the advantages of the DMM for 
'domain-disordered' structures. In $\S 4$ we demonstrate the applicability of the new calculation scheme and discuss the results of some two-dimensional model calculations. In the Appendix we outline the correlation-reduction scheme used in the calculation procedure of $\S 2$.

\section{The matrix method}

The basic idea of the matrix method is that defects occur independently in a chain (leading to a simple Markov chain) or that defects interact only locally (which gives a higher-order Markov chain). The MM can thus be looked upon as a stochastic process. Indeed it is the simplest among such processes: a Markov process of finite order. Correspondingly, the basic physical property of the MM is the geometrical correlation function, which simply is an effect of multiplying transfer probabilities. The consequence of this exponential decay of the correlation is that the reflection profiles in the $\mathrm{MM}$ are always Lorentzians or sums of Lorentzians.

We briefly review the MM to elucidate the relation to the domain statistics introduced in $\S 2$. We start with an $N \times N$ correlation matrix $P=P_{m n}$ whose elements $p_{m n}$ give the conditional probabilities for the event 'configuration $m$ follows configuration $n$ ' given the configuration $n$ at some point on the chain. Let $p_{n}$ be the probability for configuration $n$ alone and $p_{m n}(l)$ the conditional probability of configuration $m$ being followed by configuration $n$ at a distance $l$ provided the configuration $n$ is given at some fixed point on the chain. We thus get the socalled a priori $\left(p_{n}\right)$ and a posteriori probabilities $\left[p_{m n}=p_{m n}(1)\right]$ as introduced by Jagodzinski (1949) and Kakinoki \& Komura (1952) and which, respectively, correspond to the stationary and the transfer probabilities in the theory of stochastic processes (see, for example, Iosifescu, 1980; Iranpour \& Chacon, 1988).

Now with the joint probabilities $P(m, n, l)$, which give the probabilities for two configurations $m$ and $n$ at distance $l$, we get a set of equations:

the multiplication (Chapman-Kolmogoroff) equation

$$
\begin{aligned}
P_{m n}(l)=\left(P^{l}\right)_{m n} & =(P \ldots P)_{m n} \\
& =\sum_{k_{1}} \ldots \sum_{k_{l-1}} P_{m k_{1}} P_{k_{1} k_{2}} \ldots P_{k_{l-1} n} ;
\end{aligned}
$$

the starting condition

$$
p_{m n}(0)=\delta_{m n},
$$

where $\delta_{m n}$ is the Kronecker delta; the normalization conditions

$$
\sum_{m} p_{m n}(l)=1, \quad \sum_{n} p_{n}=1
$$

and

$$
\begin{aligned}
P(m, n, l) & =p_{m} p_{m n}(l)=p_{n} p_{n m}(-l) \\
& =P(n, m,-l) .
\end{aligned}
$$

With vanishing long-range order we further have

$$
\lim _{l \rightarrow \infty} p_{m n}(l)=p_{n} \text {. }
$$

Equation (1.5) defines the a priori probabilities as the eigenvectors of the correlation matrix to its maximal left eigenvalue 1. Equations (1.1)-(1.5) set up the usual conditions for an ergodic chain. We note, however, that these relations hold for a sequence of domains as well. The difference is that the distance $l$ is then the number of domain boundaries between two domains $m$ and $n$.

With (1.1)-(1.5) and the structure factors $F_{n}$ for the configurations $n$ the calculation of the intensity distribution is straightforward. All possible configurations have to be summed up - respecting all pairwise transfer probabilities. The phase factors according to the displacement between neighbouring cells are treated by complex transfer probabilities $p_{m n}^{\prime}(l)$ introduced via the identity

$$
\begin{aligned}
\left\langle F_{0} F_{l}^{*}\right\rangle & =\sum_{m} \sum_{n} p_{m} p_{m n}(l) F_{m} F_{n}^{*} \\
& =\sum_{m} \sum_{n} p_{m} p_{m n}^{\prime}(l) .
\end{aligned}
$$

The intensity is now (Wilson, 1942; Jagodzinski, 1949)

$I(k)=\sum_{l=-(N-1)}^{N-1}(N-|l|)\left\langle F_{0} F_{l}^{*}\right\rangle \exp (-i k l a)$,

where $a$ is the cell constant, $l$ is the cell index and $k$ is the projection of the scattering vector onto the disorder direction. If $P^{\prime}$ (with entries $p_{m n}^{\prime}$ ) is diagonalizable, a simple procedure gives

$$
\begin{aligned}
\left(P^{\prime \prime}\right)_{m n} & =\left(U^{-1}\left(\begin{array}{ccc}
\lambda_{1}^{\prime} & 0 & \ldots \\
0 & \lambda_{2}^{\prime} & \ldots \\
\ldots & \ldots & \lambda_{N}^{\prime}
\end{array}\right) U\right)^{l} \\
& =U^{-1}\left(\begin{array}{ccc}
\lambda_{l}^{\prime l} & 0 & \ldots \\
0 & \lambda_{2}^{\prime l} & \ldots \\
\ldots & \ldots & \lambda_{N}^{\prime \prime}
\end{array}\right) U
\end{aligned}
$$

and the final result is obtained from this by forming a geometric series. Ignoring finite size effects, it reads (Jagodzinski, 1949; Jagodzinski, Moritz \& Wolf, 1978)

$$
\begin{aligned}
I(k)= & \sum_{i=1}^{N} A_{i}\left(1-\rho_{i}^{2}\right) /\left[1-2 \rho_{i} \cos \left(k-\varphi_{i}\right)+\rho_{i}^{2}\right] \\
& -2 B_{i}\left[\rho_{i} \sin \left(k-\varphi_{i}\right)\right] \\
& \times\left[1-2 \rho_{i} \cos \left(k-\varphi_{i}\right)+\rho_{i}^{2}\right]^{-1} .
\end{aligned}
$$


The coefficients $A_{i}$ and $B_{i}$ are determined by the complex components of a third-rank tensor $C_{m n i}$ :

$$
\begin{gathered}
A_{i}(k)=\operatorname{Re} C_{i}(k), \quad B_{i}(k)=\operatorname{Im} C_{i}(k), \\
C_{i}(k)=\sum_{m} \sum_{n} C_{m n i} F_{m}(k) F_{n}^{*}(k)
\end{gathered}
$$

and the $C_{m n i}$ themselves are determined from the set. of equations

$$
p_{m} p_{m n}^{\prime}(l)=\sum_{i=1}^{N} C_{m n i} \lambda_{i}^{\prime \prime}
$$

The diffracted intensity is thus determined by the coefficients $\rho_{i}$ and $\varphi_{i}$, which, respectively, are the moduli and phases of the eigenvalues of the phaseextended matrix $P_{m n}^{\prime}$,

$$
\lambda_{i}^{\prime}=\rho_{i} \exp \left(i \varphi_{i}\right) \quad \text { with } \operatorname{det}\left(\lambda^{\prime} E-P^{\prime}\right)=0 .
$$

Following Jagodzinski (1949), (1.8) can be readily interpreted. The total profile of the (Markov) disordered structure is a sum of symmetric contributions having approximately a Lorentzian shape with amplitudes $\boldsymbol{A}_{i}$, which can be considered as generalized structure factors. In addition, asymmetric contributions (the $B_{i}$ terms) occur. Each single profile comes from one eigenvalue of the phase-extended correlation matrix $P^{\prime}$, which is now a function of the diffraction condition. The widths $\left(\rho_{i}\right)$ and positions $\left(\varphi_{i}\right)$ of all single profiles are determined by the moduli and phases of the eigenvalues $\lambda_{i}^{\prime}$ of $P^{\prime}$. We may note, however, that the calculation scheme by diagonalization only works if the right and left eigenvectors of $P^{\prime}(k)$ are identical. This is the case if, for example, $P^{\prime}$ (as a function of the scattering vector $k$ ) always remains symmetric or unitary. In general, however, this is not the case and, consequently, additional terms may arise in (1.9). For the special case of an unsymmetrically occupied four-layer model (with a $4 \times 4$ matrix $P$ ), these additional (diffuse) terms have been calculated by Kakinoki \& Komura (1952).

The MM can also be generalized to higher dimensions giving a higher-rank tensor formalism (Pimbley \& Lu, 1985a,b). This generalization, however, is nontrivial and plagued by the problem of getting a high number of correlation terms. A correct result is then difficult to obtain and so far has only been found for special cases. Study is usually restricted to either a 'scaling' (self-similarity) or the 'factorization limit' (where both disorder directions are modelled independently and the two-dimensional Markov chain is a product of two independent onedimensional chains).

\section{The domain matrix method}

In $\S 1$ we have seen that the matrix method works as a Markov model that is exactly solvable by a diagonalization procedure. We now go over to domain statistics and replace the occupation of cells by the occupation of domains of a statistical variable length. Formally, we can now write

$$
\left[p_{n}\right] \rightarrow\left[p_{\alpha}, v_{\alpha}(n)\right],
$$

which means that we 'blow up' our set of statistical parameters (observables) from cell-occupation $\left(p_{n}\right)$ to domain-occupation factors ( $p_{\alpha}$ for a domain of type $\alpha$ ) and domain-length distribution functions [ $v_{\alpha}(n)$ for a type $\alpha$ domain].

A pictorial representation of the new model is given in Fig. 1. Additional disorder parameters are now the domain-length distribution functions. As Fig. 1 shows, we now have lost the positional information of the $k$ th domain because, given the first domain, the $k$ th begins at some a priori unknown coordinate $r_{k} . r_{k}$ is now a function of the lengths of the domains between domain 1 and domain $k$. This is in contrast to cell statistics where the $k$ th cell always begins at $x_{k}=k a$. We thus see that domain statistics differs significantly from cell statistics. The difference has essentially two effects. Firstly, we do not now necessarily start from a fixed mean lattice. Secondly, as the outcome will show, the solution becomes doubled instead of simply recursive.

Now (see Fig. 1) let the whole structure consist of $K$ domains where the $k$ th domain consists of $n_{k}$ cells with structure factors $F_{k}$. To every domain $k$ one domain type $\alpha$ is assigned and the structure factor $F_{k}$ depends only on the type of the considered domain: $F_{k}=F_{\alpha}$ for some $k, \alpha$. Here we assume that the whole structure is stacked from domains of a finite number of types, the types being of varying lengths and following one another with given transition probabilities. No assumptions have been made about the lattice constants within the domains or about the distance vectors between domains. In the case of a rough surface, as will be discussed below, there may

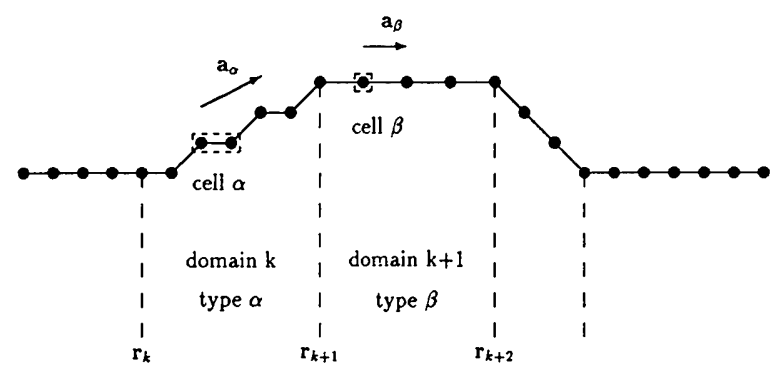

Fig. 1. Diagram of the DMM. Every domain consists of (threedimensional) cells and the domains are arranged one-dimensionally. Domain stacking is determined by $p_{\alpha \beta}$ where $\alpha, \beta$ are domain types. Every domain $k$ is of some domain type $\alpha=\alpha(k)$. No overall (global) lattice is assumed; each domain can have its own (local) lattice. The spacing, orientation, point group etc. may differ from domain to domain. Statistical parameters are the type a priori probability $p_{\alpha}$, the type a posteriori probability $p_{\alpha \beta}$ and the type length-distribution function $v_{\alpha}(n)$. For further details, refer to text $(\S 2)$. 
exist flat terraces separated by steps of different height and regularly stepped faces with varying mean orientation.

The lengths of the domains of type $\alpha$ are now described by a set of domain-length distribution functions $v_{\alpha}\left(n_{k}\right)$ that depend only on the types of the domains: $v_{\alpha}\left(n_{k}\right)=v_{\alpha}(n)$. The sequence of neighbouring domains is described by a correlation (transfer) matrix $p_{\alpha \beta}$, which we postulate to depend only on domain types as well. Note here the difference between domain-type transfer $\left(p_{\alpha \beta}\right)$ and cell-transfer $\left(p_{m n}\right)$ statistics $(\$ 1)$. In the domain-type transfer probability, as described here, interactions between neighbouring domains are considered, allowing, for example, exclusions in the domain sequence; the length of the domains, however, can vary. In cell statistics, all cells have the same (unit) length.

The calculation is now performed by a rigorous statistical treatment of all pairwise interference terms. For this we construct the autocorrelation (Patterson) function: $P(\mathbf{r})=\rho(\mathbf{r}) * \rho(-\mathbf{r})$, which gives all contributions within single-scattering (kinematical) theory. In the following, $*$ denotes convolution and the scattering vector is denoted $\mathbf{k}$. Now let $\rho_{k}(\mathbf{r})$ denote the density function of one cell within the lattice of the $k$ th domain. The electron density in the $k$ th domain is then

$$
D_{k}(\mathbf{r})=\sum_{n=1}^{n_{k}} \delta\left(\mathbf{r}-n \mathbf{a}_{k}\right) * \delta\left(\mathbf{r}-\mathbf{r}_{k}\right) * \rho_{k}(\mathbf{r}),
$$

where $\delta$ is the Dirac delta function and the Patterson function of the whole structure is

$$
\begin{aligned}
P(\mathbf{r})= & \sum_{k, k^{\prime}} D_{k}(\mathbf{r}) * D_{k^{\prime}}(-\mathbf{r}) \\
= & \sum_{k, k^{\prime}=1}^{K}\left\{\delta\left[\mathbf{r}-\left(\mathbf{r}_{k}-\mathbf{r}_{k^{\prime}}\right)\right] * \rho_{k}(\mathbf{r}) * \rho_{k^{\prime}}(-\mathbf{r})\right\} \\
& *\left\{\sum_{n=1}^{n_{k}} \sum_{m=1}^{n_{k^{\prime}}} \delta\left[\mathbf{r}-\left(n \mathbf{a}_{k}-m \mathbf{a}_{k^{\prime}}\right)\right]\right\}
\end{aligned}
$$

Now the $k$ th domain $D_{k}$ is of some type $\alpha$. We therefore can alternatively sum up (2.2) over domain types,

$$
\begin{aligned}
P(\mathbf{r})= & \sum_{\alpha \alpha^{\prime}}\left\{\rho_{\alpha}(\mathbf{r}) * \rho_{\alpha^{\prime}}(-\mathbf{r})\right. \\
& * \sum_{(k \rightarrow \alpha)=1}^{K} \sum_{\left(k^{\prime} \rightarrow \alpha^{\prime}\right)=1}^{K} \delta\left[\mathbf{r}-\left(\mathbf{r}_{k}-\mathbf{r}_{k^{\prime}}\right)\right] \\
& \left.* \sum_{n=1}^{n_{k}} \sum_{m=1}^{n_{k^{\prime}}} \delta\left[\mathbf{r}-\left(n \mathbf{a}_{\alpha}-m \mathbf{a}_{\alpha^{\prime}}\right)\right]\right\} .
\end{aligned}
$$

Here we have used the fact that $\rho_{k}(\mathbf{r})=\rho_{\alpha}(\mathbf{r})$ if the $k$ th domain is of type $\alpha$. The symbol $k \rightarrow \alpha$ means $k$ provided the type of $k$ is $\alpha$.
Next we introduce joint probability densities and make use of the mathematical identity

$\sum_{(k \rightarrow \alpha)=1}^{K} \sum_{\left(k^{\prime} \rightarrow \alpha^{\prime}\right)=1}^{K} 1=\sum_{k=1}^{K} \sum_{k^{\prime}=1}^{K} p\left(k \rightarrow \alpha, k^{\prime} \rightarrow \alpha^{\prime}\right)$.

The (domain) joint probability densities can now be expressed by our model, which allows only influences between neighbouring domains. We use the (homogeneous) Markov-chain properties for domain-type statistics,

$p\left(k \rightarrow \alpha, k+l \rightarrow \alpha^{\prime}\right)=p_{\alpha} p_{\alpha \alpha^{\prime}}(l) \quad$ independent of $k$

and the reciprocity conditions

$$
\begin{aligned}
& p\left(k \rightarrow \alpha, k^{\prime} \rightarrow \alpha^{\prime}\right)=p\left(k^{\prime} \rightarrow \alpha^{\prime}, k \rightarrow \alpha\right), \\
& p\left(k \rightarrow \alpha, k \rightarrow \alpha^{\prime}\right)=\delta_{\alpha \alpha^{\prime}} .
\end{aligned}
$$

The Markov-chain index $l$ now counts the number of domain boundaries between the considered domain pair. Keeping the analogy between domain and cell statistics in mind we can split $(2.3)$ into a self-domain Patterson contribution $P_{s}(\mathbf{r})$ and a distinct-domain Patterson contribution $P_{d}(\mathbf{r})$. With (2.4)-(2.6) we get

$P_{s}(\mathbf{r})=\sum_{\alpha} \rho_{\alpha} * \rho_{\alpha}^{-} * \sum_{k=1}^{K} \sum_{n=1}^{n_{k}} \sum_{m=1}^{n_{k}} p_{\alpha} \delta\left[\mathbf{r}-\left(n \mathbf{a}_{\alpha}-m \mathbf{a}_{\alpha^{\prime}}\right)\right]$

and

$$
\begin{aligned}
P_{d}(\mathbf{r})= & \sum_{\alpha, \alpha^{\prime}} \rho_{\alpha} * \rho_{\alpha^{\prime}}^{-} \\
& * \sum_{l=1}^{K}\left\{p_{\alpha} p_{\alpha \alpha^{\prime}}^{(l)} \sum_{k=1}^{K} \delta\left[\mathbf{r}-\left(\mathbf{r}_{k}-\mathbf{r}_{k+l}\right)\right]\right. \\
& * \sum_{n=1}^{n_{k}} \sum_{m=1}^{n_{k+1}} \delta\left[\mathbf{r}-\left(n \mathbf{a}_{\alpha}-m \mathbf{a}_{\alpha^{\prime}}\right)\right] \\
& +p_{\alpha^{\prime}} p_{\alpha^{\prime} \alpha}^{(l)} \sum_{k=1}^{K} \delta\left[\mathbf{r}-\left(\mathbf{r}_{k}-\mathbf{r}_{k-l}\right)\right] \\
& \left.* \sum_{n=1}^{n_{k}} \sum_{m=1}^{n_{k-1}} \delta\left[\mathbf{r}-\left(n \mathbf{a}_{\alpha}-m \mathbf{a}_{\alpha^{\prime}}\right)\right]\right\}
\end{aligned}
$$

where we have dropped the $\mathbf{r}$ arguments in the first terms and have defined $\rho_{\alpha}^{-}(\mathbf{r})=\rho_{\alpha}(-\mathbf{r})$. After Fourier transformation we get the intensity as

$$
I(\mathbf{k})=I_{s}(\mathbf{k})+I_{d}(\mathbf{k}),
$$

with the self-domain (or zero boundary, $l=0$ ) term

$$
\begin{aligned}
I_{s}(\mathbf{k})= & K \sum_{\alpha} F_{\alpha} F_{\alpha}^{*} p_{\alpha} \sum_{n} v_{\alpha}(n) \\
& \times F_{\mathrm{DD}}(n, n, \alpha, \alpha, 0 ; \mathbf{k})
\end{aligned}
$$


and the distinct-domain (or nonzero boundary, $l>0$ ) term

$$
\begin{aligned}
I_{d}(\mathbf{k})= & K \sum_{\alpha \alpha^{\prime}} F_{\alpha} F_{\alpha^{\prime}}^{*} \sum_{l=1}^{K} p_{\alpha} p_{\alpha \alpha^{\prime}}^{(l)} \sum_{m n} v_{\alpha}(m) \\
& \times v_{\alpha^{\prime}}(n) F_{\mathrm{DD}}\left(m, n, \alpha, \alpha^{\prime}, l ; \mathbf{k}\right) \\
& \times\left.\left\langle F_{\mathrm{DB}}\left(m, n, \alpha, \alpha^{\prime}, l ; \mathbf{k}\right)\right\rangle_{k}\right|_{\alpha^{\prime} n} \\
& +K \sum_{\alpha \alpha^{\prime}} F_{\alpha^{\prime}} F_{\alpha}^{*} \sum_{l=1}^{K} p_{\alpha^{\prime}} p_{\alpha^{\prime} \alpha}^{(l)} \\
& \times \sum_{m n} v_{\alpha}(m) v_{\alpha^{\prime}}(n) F_{\mathrm{DD}}\left(n, m, \alpha^{\prime}, \alpha, l ; \mathbf{k}\right) \\
& \times\left.\left\langle F_{\mathrm{DB}}\left(n, m, \alpha^{\prime}, \alpha, l ; \mathbf{k}\right)\right\rangle_{k}\right|_{\substack{\alpha^{\prime} n \\
\alpha^{\prime}}}
\end{aligned}
$$

Here we have replaced the (domain) configurational $k$ sums in (2.7) by $K$ times their configurational averages and introduced a domain-domain term

$$
\begin{aligned}
& F_{\mathrm{DD}}\left(m, n, \alpha, \alpha^{\prime} ; \mathbf{k}\right) \\
& \quad=\sum_{n^{\prime}=1}^{n} \sum_{m^{\prime}=1}^{m} \exp \left[i \mathbf{k} \cdot\left(n^{\prime} \mathbf{a}_{\alpha}-m^{\prime} \mathbf{a}_{\alpha^{\prime}}\right)\right]
\end{aligned}
$$

and a domain-boundary (or domain-distance) term

$$
\begin{aligned}
& \left.\left\langle F_{\mathrm{DB}}\left(m, n, \alpha, \alpha^{\prime} ; \mathbf{k}\right)\right\rangle_{k}\right|_{\substack{\alpha m \\
\alpha^{\prime} n}} \\
& \quad=\left.\left\langle\exp \left[i \mathbf{k} \cdot\left(\mathbf{r}_{k}-\mathbf{r}_{k+l}\right)\right]\right\rangle_{k}\right|_{\substack{k \rightarrow \alpha, n_{k}=m \\
k+l \rightarrow \alpha^{\prime}, n_{k+1}=n}} .
\end{aligned}
$$

We see that we get a constricted average $\left.\langle\ldots\rangle_{k}\right|_{\alpha^{\prime} n}$ as a result of summing up domain types (instead of domains) in (2.3). The restriction originates from the fact that a pair of non-neighbouring domains is correlated with all pairs of neighbouring domains (of other types) via the domain transfer matrix. The logical step from (2.7) to (2.8)/(2.9) is worked out in Lemma 1 of the Appendix. Equation (2.8) should be compared with the more familiar result for a cell statistic ( $L \gg 1$ cells)

$$
\begin{aligned}
I(\mathbf{k})= & I_{s}(\mathbf{k})+I_{d}(\mathbf{k}) \\
= & |\langle F(\mathbf{k})\rangle|^{2} \sum_{\mathbf{g}} \delta(\mathbf{k}-\mathbf{g})+L \sum_{i=1}^{L-1}\left\langle\left[F_{0}(\mathbf{k})-\langle F(\mathbf{k})\rangle\right]\right. \\
& \left.\times\left[F_{l}^{*}(\mathbf{k})-\left\langle F^{*}(\mathbf{k})\right\rangle\right]\right\rangle \exp (i l \mathbf{k} \cdot \mathbf{a}) .
\end{aligned}
$$

Let us briefly discuss the meaning of the domaindomain and the domain-distance term. $F_{\mathrm{DD}}$ is the cross term of a pair of domains of given types $\alpha$ and $\alpha^{\prime}$ - provided both domains begin at the same point in real space. However, since the two domains are shifted, a second term, $F_{\mathrm{DB}}$, arises that allows for this shifting.

The total diffracted intensity is now the sum of $(2.8 a)$ and $(2.8 b)$. Compactly written and after a little calculation, it reads (with the simplified notation

$$
\begin{aligned}
&\left.\underset{\alpha^{\prime}}{\alpha^{\prime}} \text { for }\left.\right|_{\substack{\alpha m n \\
\alpha^{\prime} n}}\right) \\
& I(\mathbf{k})= K \sum_{\alpha}\left[F_{\alpha}(\mathbf{k}) F_{\alpha}^{*}(\mathbf{k}) \sum_{n=1}^{n_{\alpha}} v_{\alpha}(n) F_{\mathrm{DD}}(n, n, \alpha, \alpha ; \mathbf{k})\right] \\
&+K \sum_{\alpha \alpha^{\prime}} \sum_{l=1}^{K} p_{\alpha} p_{\alpha \alpha^{\prime}}^{(l)} \sum_{m=1}^{n_{\alpha}} \sum_{n=1}^{n_{\alpha^{\prime}}} v_{\alpha}(m) v_{\alpha^{\prime}}(n) \\
& \times 2 \operatorname{Re}\left[F_{\alpha}(\mathbf{k}) F_{\alpha^{\prime}}^{*}(\mathbf{k}) F_{\mathrm{DD}}\left(m, n, \alpha, \alpha^{\prime} ; \mathbf{k}\right)\right. \\
&\left.\times\left.\left\langle F_{\mathrm{DB}}\left(m, n, \alpha, \alpha^{\prime}, l ; \mathbf{k}\right)\right\rangle_{k}\right|_{\alpha^{\prime}}\right] .
\end{aligned}
$$

With (2.10) we have now derived a closed-form solution for the diffracted intensity of an array of onedimensionally arranged domains in its most general form. As already mentioned, the main advantage of this solution is that the domain-length distributions $v_{\alpha}(n)$ are entirely decoupled from the inter-domain correlations determined by $p_{\alpha \beta}$.

To proceed with the calculation of $\left.\left\langle F_{\mathrm{DB}}\right\rangle_{k}\right|_{\alpha^{\prime}}$ we must now tackle the previously mentioned correlation problem: despite the decoupling of the domain lengths $v_{\alpha}(n)$ from the domain transfer probabilities $p_{\alpha \beta}$ the domain-boundary (domain-distance) term $F_{\mathrm{DB}}$ is heavily correlated with all possible domains lying between the first and the last domain in the domain-domain term $F_{\mathrm{DD}}$. It is because of this correlation that the domain-domain term $F_{\mathrm{DD}}$ and the domain-distance term $F_{\mathrm{DB}}$ cannot be averaged independently (see Lemma 2 of the Appendix). In the work of Houston \& Park $(1970,1971)$ this correlation has been neglected - a simplification that in general is not allowed.

We now give the correct calculation of the averaged domain-boundary factor $\left\langle\left. F_{\mathrm{DB}}\right|_{\alpha^{\prime}}\right.$. The method we use is based on the following idea. Mapping $\left.\left\langle F_{\mathrm{DB}}(l)\right\rangle\right|_{\alpha^{\prime}}$ to $\left.\left\langle F_{\mathrm{DB}}(l-1)\right\rangle\right|_{\alpha^{\prime}}$ gives the same correlation problem with a reduced $l$. If we therefore iterate that mapping procedure we will finally arrive at the trivial case $l=1$ for which the domains are neighbours. Then, of course, we totally get rid of the constraint $\left.\right|_{\alpha^{\prime}}$ - because the origin of two neighbouring domains is simply shifted by the length of one of the domains. As shown in detail in Lemma 3 of the Appendix, the final result from this idea can be brought into the recursion formula $(l \geq 1)$

$$
\begin{aligned}
& \left.\left\langle F_{\mathrm{DB}}\left(m, n, \alpha, \alpha^{\prime}, l ; \mathbf{k}\right)\right\rangle_{k}\right|_{\alpha^{\prime}} \\
& =\sum_{n^{\prime} \gamma^{\prime}} v_{\gamma^{\prime}}\left(n^{\prime}\right)\left[p_{\alpha \gamma^{\prime}}^{(l-1)} p_{\gamma^{\prime} \alpha^{\prime}} / p_{\alpha \alpha^{\prime}}^{(l)}\right] \exp \left(-i n^{\prime} \mathbf{k} \cdot \mathbf{a}_{\gamma^{\prime}}\right) \\
& \quad \times\left.\left\langle F_{\mathrm{DB}}\left(m, n^{\prime}, \alpha, \gamma^{\prime}, l-1 ; \mathbf{k}\right)\right\rangle_{k}\right|_{\gamma^{\prime}}
\end{aligned}
$$


with the initial condition $(l=1)$

$$
\begin{aligned}
\left\langle F_{\mathrm{DB}}\left(m, n, \alpha, \alpha^{\prime}, 1 ; \mathbf{k}\right)\right\rangle_{k} & =\exp \left(-i m \mathbf{k} \cdot \mathbf{a}_{\alpha}\right) \\
& =\exp \left(i n \mathbf{k} \cdot \mathbf{a}_{\alpha^{\prime}}\right) .
\end{aligned}
$$

We have now solved the domain-disordering problem quite generally. With (2.10) and (2.11) we have obtained a partly closed-form, partly recursive expression for the diffracted intensity of an arbitrary one-dimensionally disordered three-dimensional domain structure.

Comparing (2.10) and (2.11) with the matrixmethod solution (1.9), we see that the price of domain statistics - compared to cell statistics - is a more complicated result. Instead of the single recursive equations $(1.6) /(1.7)$ we now get a double recursive solution $(2.10) /(2.11)$.

Let us now discuss our solution, for one particular limiting case: a pure cell disorder, which means that all domains have unit length. Then introducing (with suppressed $\mathbf{k}$ )

$$
p_{\substack{\alpha \alpha^{\prime} \\ m n}}^{\prime}(l)=p_{\alpha \alpha^{\prime}}(l) F_{\mathrm{DB}}\left(m, n, \alpha, \alpha^{\prime}, l\right)
$$

we first get from (2.11), because $v_{\alpha}(n)=\delta_{1, n}$,

$$
p_{\substack{\alpha \alpha^{\prime} \\ m n}}^{\prime}(l)=\sum_{\gamma^{\prime}} p_{\substack{\alpha \\ y^{\prime}}}^{\prime}(l-1) p_{\substack{\gamma^{\prime} \alpha^{\prime} \\ 1}}^{\prime}(1),
$$

which has the form of a matrix multiplication. Inserted in (2.10), the diffracted intensity $I$ can now be simplified to give ( $\mathbf{k}$ suppressed)

$$
\begin{aligned}
I= & \sum_{\alpha \alpha^{\prime}} p_{\alpha} \sum_{m n} v_{\alpha}(m) v_{\alpha^{\prime}}(n) F_{\alpha} F_{\alpha^{\prime}}^{*} \\
& \times F_{\mathrm{DD}}\left(m, n, \alpha, \alpha^{\prime}\right) \sum_{l} p_{\substack{\alpha \alpha^{\prime} \\
m n}}^{\prime}(l) \\
= & \sum_{\alpha \alpha^{\prime}} p_{\alpha} F_{\alpha} F_{\alpha^{\prime}}^{*} F_{\mathrm{DD}}\left(1,1, \alpha, \alpha^{\prime}\right) \sum_{\substack{l \\
p_{\alpha \alpha^{\prime}}(l) \\
=}} \sum_{\alpha \alpha^{\prime}} p_{\alpha} F_{\alpha} F_{\alpha^{\prime}}^{*} F_{\mathrm{DD}}\left(1,1, \alpha, \alpha^{\prime}\right) \\
& \times \sum_{l} F_{\mathrm{DB}}\left(1,1, \alpha, \alpha^{\prime}, l\right) p_{\alpha \alpha^{\prime}}(l),
\end{aligned}
$$

which, because $\mathbf{a}_{\alpha}=\mathbf{a}, \quad F_{\mathrm{DD}}\left(1,1, \alpha, \alpha^{\prime}\right)=$ $\exp \left[i \mathbf{k} \cdot\left(\mathbf{a}_{\alpha}-\mathbf{a}_{\alpha^{\prime}}\right)\right]$ and $F_{\mathrm{DB}}\left(1,1, \alpha, \alpha^{\prime}, l\right)=$ $\exp \left(-i \mathbf{k} \cdot \mathbf{a}_{\alpha}\right)$, is exactly $(1.7)(N \gg 1)$ with a phaseextended correlation matrix

$$
p_{\alpha \alpha^{\prime}}^{\prime}=F_{\alpha} F_{\alpha^{\prime}}^{*} p_{\alpha \alpha^{\prime}} \text {. }
$$

We therefore find that the DMM reduces to the MM if all domains are (equal-spaced) cells. This, of course, is a necessary condition. It should be noted, however, that in all other cases [if $v_{\alpha}(n) \neq \delta_{1, n}$ ] the complicated interconnectivity of all correlation terms in (2.10) prevents us from getting a simple matrix solution. This in turn means that the DMM is a true generalization of the MM and therefore offers new perspectives.

\section{Discussion: domain disordering}

We now discuss some examples of domain disordering where the MM is not well suited to describe the reflection profiles. Cases of disordered crystal surfaces will be mentioned since we will later apply the method to such problems. The method may also be applied to bulk crystals with planar faults. As an initial example we consider a surface endowed with an atomic step structure. Monoatomic, biatomic, triatomic etc. steps may occur simultaneously on the surface. Multiple-height steps of up to three or four layers have been reported from many surface systems with various methods (STM, LEED, RHEED). Biatomic steps have been observed in the $\mathrm{Si}(001)$ surface (Aspnes \& Ihm, 1986; Wierenga, Kubby \& Griffith, 1987), where they dominate over monoatomic steps if a critical tilt angle of about $2.5-5^{\circ}$ is exceeded. Steps of larger height are also frequently observed. They are intimately related with surface roughening and adsorbate growth mechanisms. Adsorbate-induced faceting is also observed in many surfaces (see, for example, Boulliard \& Sotto, 1986; Barthes-Labrousse, 1986).

Apart from step-terrace and facet structures, a number of other types of domain structures may occur both on surfaces and in the bulk. Here we only give two examples: the recent discovery of long-range correlations in the low-coverage $\mathrm{O} / \mathrm{Cu}(110)-(2 \times 1)$ structure (Kern et al., 1991) and the observation of long-range step-step correlations on a well oriented $\mathrm{Si}(100)$ surface by high-resolution $\mathrm{X}$-ray grazingincidence diffraction (Renaud, Fuoss, Bevk \& Freer, 1992).

For many such kinds of domain disordering a model description by the MM is not possible in practice since the correlation matrix becomes extremely large. The reduction of a huge matrix with a large number of disorder parameters to a small number of reasonable physical quantities is not only a tedious procedure but also in many cases is not unique. In the case of longer correlations, the disorder problem is therefore more appropriately solved by introducing domains instead of cells and calculating the Patterson function from its distributions (the DMM).

If we compare the new result [(2.10), (2.11)] with the MM (1.9), the missing analyticity in the solution of the DMM is, of course, a clear disadvantage. We believe, however, that this disadvantage is more than compensated for by both the gain of a more general model and the elimination of the matrix inflation problem. The big advantage of the DMM is that long domains can be calculated as easily as short ones. By a suitable choice of the domains, there is no problem of a matrix inflation in the DMM.

A number of additional remarks may be made. The first is related to Adlhart's model. In this model (Adlhart, 1981), a quite general (periodic) domain 
sequence is considered that allows a kinematical exact and analytical solution in terms of a geometrical series. Due to the assumed periodic stacking, however, all domains are equally probable in this model - an unrealistic assumption for structures with elements of differing probabilities. Furthermore, the averaging over domains becomes difficult in Adlhart's model if there are more than two domain types. The DMM, in contrast, is not plagued by these problems. It also allows the description of structures with elements of differing probabilities and is thus a generalization from Adlhart's cyclic model to a not necessarily cyclic one.

Secondly, we have restricted ourselves to kinematical theory. This is suitable for X-ray and neutron diffraction. For electron diffraction (LEED, RHEED), multiple-scattering processes arise and a kinematic evaluation of intensities is generally not possible. In the case of LEED, however, multiple scattering is short ranged and the DMM can be used to simulate multiple-scattering processes since it allows the introduction of fringes at domain boundaries differing in scattering factor.

A third point concerns convergence problems. In practice no problems arise. Except at the Bragg points, the sums in the DMM converge rapidly especially if they are convoluted with a response function to respect instrumental broadening. This convolution, when included in a computer program, has the effect of introducing a damping term in the recursion equation (2.11) and thus guarantees the numerical stability of our algorithm.

\section{Applications to surfaces: steps and facets}

We now describe some applications of the DMM. For a rough (100) surface and for different types of roughness (see Fig. 2) we have calculated some

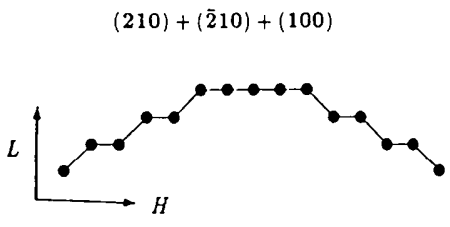

(a)

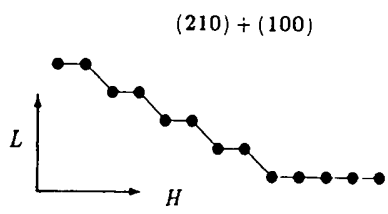

(b)

Fig. 2. Partially faceted surfaces (hill-and-valley structures), sketched schematically; (a) facets on a symmetrical surface, (b) facets on an asymmetrical (miscut) surface. diffraction profiles; the results are presented in Figs. 3-6.

In Fig. 3, a partially faceted surface has been simulated according to the hill and valley structures sketched in Fig. 2. The existence of such types of surface structures has recently been demonstrated for the thermally roughened $\mathrm{Ag}(110)$ face (Robinson, Vlieg, Hornis \& Conrad, 1991), which undergoes a roughening transition at an elevated temperature $\left(T_{R}=790 \mathrm{~K}\right)$. As a result, a characteristic and diffraction-condition-dependent shifting of satellites occurs, superposed on the specularly diffracted beam (Fig. 3).

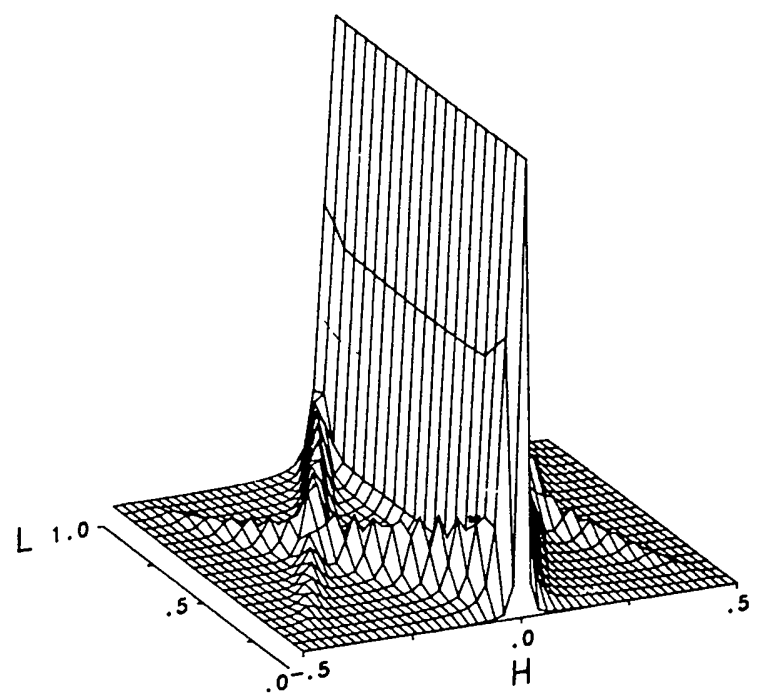

(a)

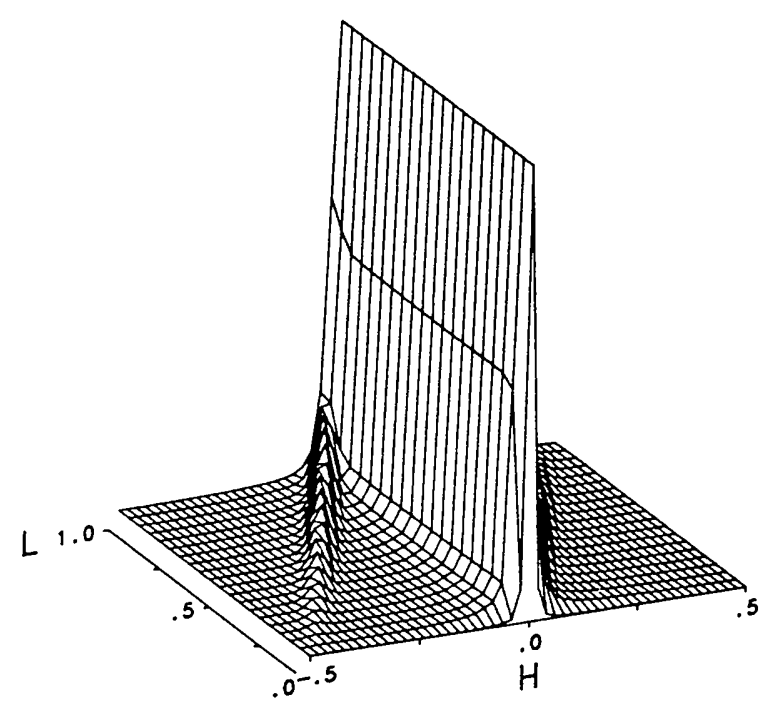

(b)

Fig. 3. Calculated intensity plots $I(H, L)$ for the hill-and-valley structures of Fig. 2; a Gaussian resolution function of FWHM = 0.05 rec. units has been assumed. $(a)(210)$ and $(\overline{2} 10)$ facets of 8 cells (three-unit steps) with (100) terraces of 20 cells (one-unit steps); (b) without the $(\overline{2} 10)$ facets. In $(b)$ the surface is miscut by $2.5^{\circ}$ (along [010]). 
Fig. 4 shows two profiles of Fig. 3(b) after convolution with a Gaussian resolution function of FWHM $=0.05$ rec. units. The diffraction conditions are $L=0.1$, close to the in-phase condition, and $L=$ 0.4 , close to the out-of-phase condition. (At the inphase condition, neighbouring terraces scatter with a phase difference of $2 \pi$; at the out-of-phase condition, with $\pi$.) As an effect of the instrumental broadening, the profiles now look asymmetric although the exact interpretation would be a superposition of two translated symmetrical profiles. With that calculation, we caution against a simple 'symmetric' or 'asymmetric' interpretation of an experimental diffraction profile. A truly asymmetric profile in general is not distinct from a superposition of several symmetric profiles.

In Fig. 5 the influence of the type of the terrace distribution function on the out-of-phase profile ( $L=$ 0.5 ) of a step-terrace structure is shown. Due to an antiphase relation across the steps, the profiles split into resolved parts if one terrace length dominates all the others [Fig. 5( $a)$, Poisson distribution]. This is the case if, for example, a random-walking adsorbate possesses a preferred island size. In the case of a geometrical distribution function the most probable size is zero and no splitting occurs [Fig. 5(b), geometrical distribution]. The theoretical profile in this case is a $\delta$ peak over a broad Lorentzian shoulder (see, for example, Wollschlaeger \& Henzler, 1989). The $\delta$ peak (which in the matrix method comes from

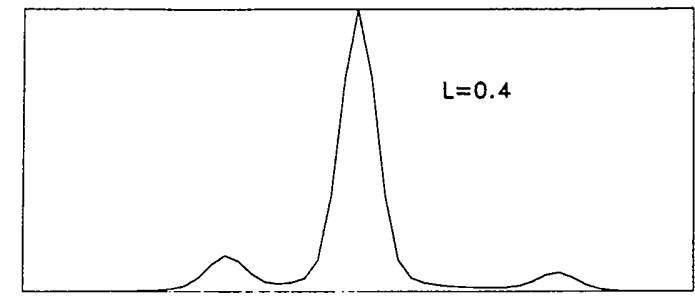

(a)

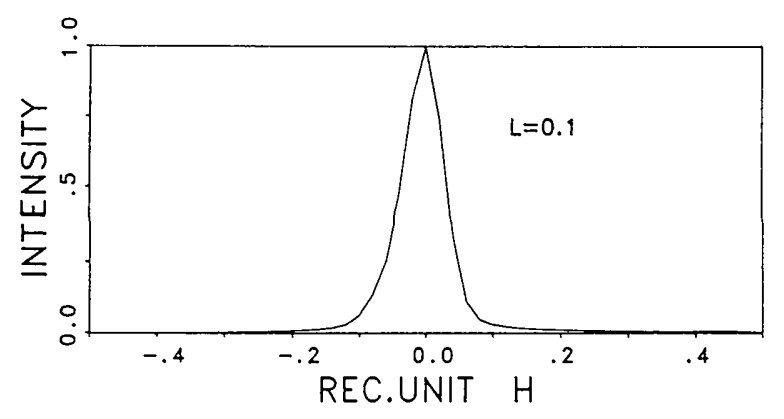

(b)

Fig. 4. Two profiles of Fig. 3(b) $(L=0.1, L=0.4)$. (a) $(210)+$ $(\overline{2} 10)+(100)$ facets, $(b)(210)+(100)$ facets. As a consequence of the assumed partial faceting of the surface the main peak looks asymmetrically distorted and is accompanied by additional (with $L$ varying) satellite peaks. an eigenvalue 1 in $P^{\prime}$, see $\S 1$ ) is here due to the missing higher atomic steps in the model, which generates a long-range phase correlation along the surface.

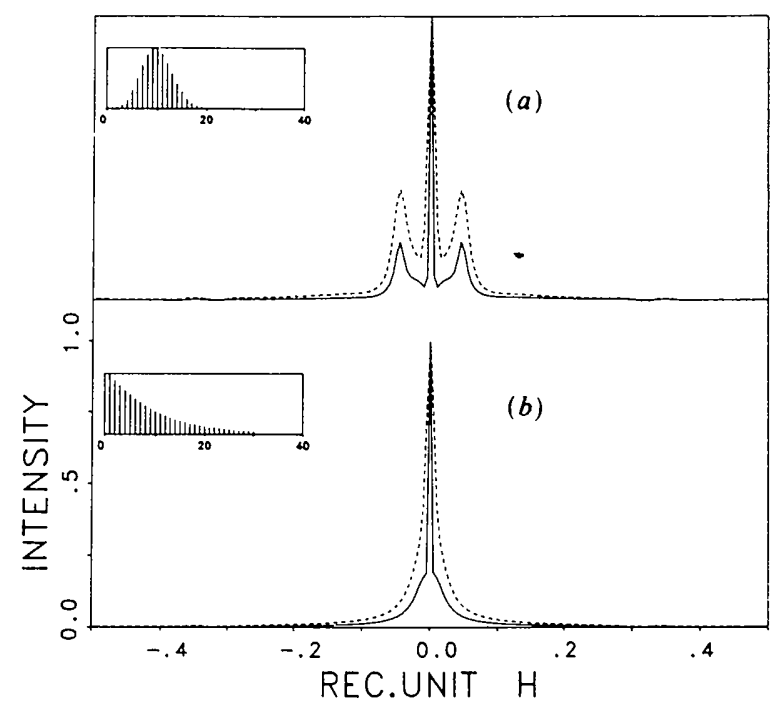

Fig. 5. Influence of the type of the terrace-width distribution on the diffraction profile of a step-terrace structure. Out-of-phase condition: $L=0.5 ;(a)$ Poisson distribution, $(b)$ geometrical distribution. Mean terrace width is 10 units. All steps have height one unit (monosteps) and are separated by terraces; double and higher atomic steps are suppressed. Continuous line: raw data. Broken line: data convoluted with a Gaussian detector function ( $F W H M=0.05$ rec. units).

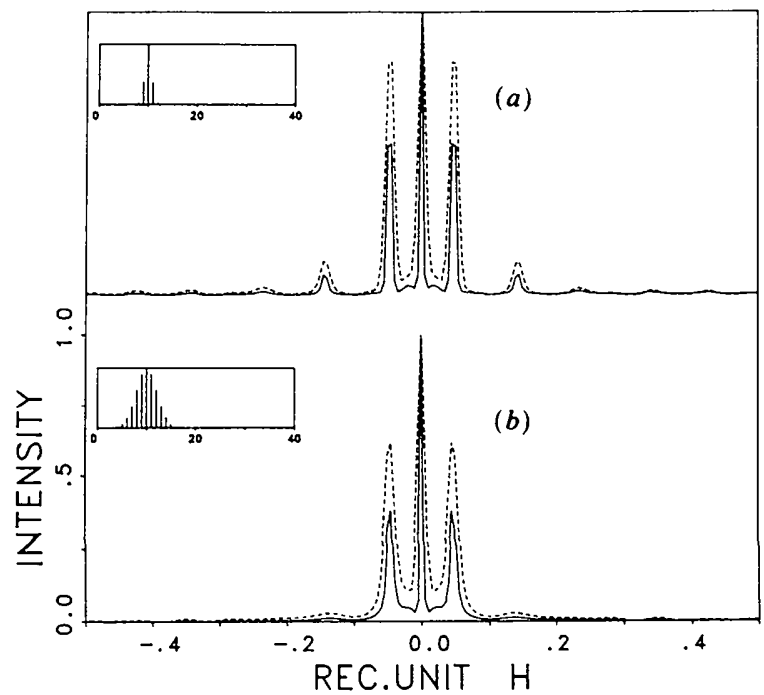

Fig. 6. Influence of the width of the terrace-width distribution on the diffraction profile of a step-terrace structure. Out-of-phase condition: $L=0.5$; Gaussian distributions ( $a$ ) of width one unit, $(b)$ of width three units. Mean terrace width is 10 units. All steps have height one unit (monosteps) and are separated by terraces; double and higher atomic steps are suppressed. Continuous line: raw data. Broken line: data convoluted with a Gaussian detector function (FWHM $=0.05$ rec. units) 
In Fig. 6 the influence of the width of the terrace distribution function is shown. A relatively sharp distribution [Fig. 6(a), Gaussian distribution with a small variance] gives more pronounced satellites than a broader one [Fig. $6(b)$, Gaussian distribution with a large variance].

From Figs. 5 and 6 we see that not only some details but even the number and form of the satellites depend on the assumed type and width of the distribution function.

\section{Concluding remarks}

We have developed a new profile-calculation scheme for domain structures with one direction of disorder. The method is more general than the matrix method although it reduces to it in the simple case of a cell statistic. A general description for the influence of the length distribution and the domain type stacking on the diffracted intensity has been gained. We have also demonstrated the advantages of the new method with some examples of surface problems. A large number of domain structures can be studied with our model.

We thank Professor M. Henzler (Hannover) and Dr E. Vlieg (Amsterdam) for fruitful discussions and interest in the DMM model. We are indebted to our co-workers (Professor D. Wolf, Dr H. L. Meyerheym, V. Jahns, J. Wever, R. Zuschke) and to Professor F. Frey for reading the manuscript. Part of this work was supported by the Deutsche Forschungsgemeinschaft (SFB 338).

\section{APPENDIX}

We prove three lemmas concerned with the statistical averaging of random variables. Lemma 1 explains the step from (2.7) to (2.8) in $\S 2$. Lemma 2 demonstrates why the domain-domain and the domain-boundary term in (2.10) cannot be averaged independently and together with Lemma 3 we get the proof of the recursion equation (2.11).

Lemma 1 (Averaging $F$ subject to constraints)

Let $F$ be a function of $\alpha$ and $\alpha^{\prime}$ and additionally of some discrete random variables $n_{k}, n_{k \pm 1}, \ldots, n_{k \pm l}$ with the respective density functions $p\left(n_{k \pm i}=n\right), i \in$ $\{0, \ldots, l\}, n \in \mathrm{N}$. The density functions $p\left(n_{k \pm i}=n\right)$ will depend - according to the sumsumption function $f: k \rightarrow \alpha-$ only on the types $\alpha$ and not on the $k$ 's themselves, i.e. for $f(k \pm i)=\alpha$ it is $p\left(n_{k \pm i}=n\right)=$ $v_{\alpha}(n)$ independent of $k$ and $i$. Then (with $\left.\right|_{\substack{\alpha \\ \alpha^{\prime}}}:=\left.\right|_{\substack{k \rightarrow \alpha \\ k \pm l \rightarrow \alpha^{\prime}}}$ )
the expectation of $F$ is

$$
\left.\left\langle F\left(n_{k}, \alpha, \alpha^{\prime}\right)\right\rangle_{k}\right|_{\alpha}=\sum_{n} v_{\alpha}(n) F\left(n, \alpha, \alpha^{\prime}\right) .
$$

Proof. By direct calculation we get

$$
\begin{aligned}
\left.\left\langle F\left(n_{k}, \alpha, \alpha^{\prime}\right)\right\rangle_{k}\right|_{\alpha^{\prime}} & =\left.\sum_{n} p\left(n_{k}=n\right) F\left(n, \alpha, \alpha^{\prime}\right)\right|_{\alpha^{\prime}} \\
& =\left.\sum_{n} p\left(n_{k}=n\right)\right|_{\alpha^{\prime}} F\left(n, \alpha, \alpha^{\prime}\right) \\
& =\sum_{n} v_{\alpha}(n) F\left(n, \alpha, \alpha^{\prime}\right) .
\end{aligned}
$$

With Lemma $1(2.8 a)$ now follows directly from

Lemma 2 (Averaging $F * G$ subject to constraints)

Let $F$ and $G$ be functions of $\alpha$ and $\alpha^{\prime}$ and additionally of some discrete random variables $n_{k}, n_{k \pm 1}, \ldots, n_{k \pm l}$ with the respective density functions $p\left(n_{k \pm i}=n\right), i \in\{0, \ldots, l\}, n \in \mathrm{N}$. As in Lemma 1 the density functions $p\left(n_{k \pm i}=n\right)$ will depend according to the sumsumption function $f: k \rightarrow$ $\alpha$ - only on types, i.e. for $f(k \pm i)=\alpha$ it is $p\left(n_{k \pm i}=n\right)=v_{\alpha}(n)$ independent of $k$ and $i$. Then (with $\left.\right|_{\alpha^{\prime}}:=\left.\right|_{\substack{k \rightarrow \alpha \\ k \pm l \rightarrow \alpha^{\prime}}}$ and $*$ representing convolution or multiplication) the expectation of $F * G$ is

$$
\begin{aligned}
& \left.\left\langle F\left(n_{k}, n_{k \pm l}, \alpha, \alpha^{\prime}\right) * G\left(n_{k}, n_{k \pm 1}, \ldots, n_{k \pm l}, \alpha, \alpha^{\prime}\right)\right\rangle_{k}\right|_{\alpha \alpha^{\prime}} \\
& =\sum_{m} \sum_{n} v_{\alpha}(m) v_{\alpha^{\prime}}(n) F\left(m, n, \alpha, \alpha^{\prime}\right) \\
& \left.\quad *\left\langle G\left(n_{k}, n_{k \pm 1}, \ldots, n_{k \pm l}, \alpha, \alpha^{\prime}\right)\right\rangle_{k}\right|_{\substack{a, n_{n}=m \\
a, n_{k \neq 1}=n}} \quad(A .2)
\end{aligned}
$$

Proof. Step by step and respecting all constraints we get

$$
\begin{aligned}
& \left.\left\langle F\left(n_{k}, n_{k \pm l}, \alpha, \alpha^{\prime}\right) * G\left(n_{k}, n_{k \pm 1}, \ldots, n_{k \pm l}, \alpha, \alpha^{\prime}\right)\right\rangle_{k}\right|_{\alpha^{\prime}} \\
& =\sum_{z_{0}} \ldots \sum_{z_{l}} p\left(n_{k}=z_{0}\right) p\left(n_{k \pm 1}=z_{l}\right) \ldots p\left(n_{k \pm l}=z_{l}\right) \\
& \times\left. F\left(z_{0}, z_{l}, \alpha, \alpha^{\prime}\right) * G\left(z_{0}, z_{1}, \ldots, z_{l}, \alpha, \alpha^{\prime}\right)\right|_{\alpha^{\prime}} \\
& =\sum_{z_{0}} \sum_{z_{l}} p\left(n_{k}=z_{0}\right) p\left(n_{k \pm l}=z_{l}\right) F\left(z_{0}, z_{l}, \alpha, \alpha^{\prime}\right) \\
& * \sum_{z_{1}} \ldots \sum_{z_{l-1}} p\left(n_{k \pm 1}=z_{1}\right) \ldots p\left(n_{k \pm(l-1)}=z_{l-1}\right) \\
& \left.G\left(z_{0}, z_{1}, \ldots, z_{l}, \alpha, \alpha^{\prime}\right)\right|_{\alpha^{\prime}} \\
& =\left[\left.\left.\sum_{m} \sum_{n} p\left(n_{k}=m\right)\right|_{\alpha^{\prime}} p\left(n_{k \pm l}=n\right)\right|_{\alpha^{\prime}} F\left(m, n, \alpha, \alpha^{\prime}\right)\right] \\
& *\left[\left.\left.\sum_{z_{1}} \ldots \sum_{z_{l-1}} p\left(n_{k \pm 1}=z_{1}\right)\right|_{\alpha^{\prime}} \ldots p\left(n_{k \pm(l-1)}=z_{l-1}\right)\right|_{\alpha^{\prime}}\right. \\
& \left.\times\left. G\left(m, z_{1} \ldots z_{l-1}, n, \alpha, \alpha^{\prime}\right)\right|_{\alpha^{\prime}}\right] \\
& =\sum_{m} \sum_{n} v_{\alpha}(m) v_{\alpha^{\prime}}(n) F\left(m, n, \alpha, \alpha^{\prime}\right) \\
& \left.*\left\langle G\left(n_{k}, n_{k \pm 1}, \ldots, n_{k \pm l}, \alpha, \alpha^{\prime}\right)\right\rangle_{k}\right|_{\substack{\alpha, n_{k}=m \\
\alpha^{\prime}, n_{k \pm l}=n}} \text {. }
\end{aligned}
$$


With Lemma 2 it is now clear that generally

$$
\left.\langle F * G\rangle_{k}\right|_{\alpha^{\prime}} \neq\left.\left.\langle F\rangle_{k}\right|_{\alpha^{\prime}} *\langle G\rangle_{k}\right|_{\alpha^{\prime}}
$$

which means that (identifying $F$ with $F_{\mathrm{DD}}$ and $G$ with $F_{\mathrm{DB}}$ of $\S 2$ ) the domain-domain and the domainboundary terms cannot be averaged independently. We further get $(2.8 b)$ from $(2.7 b)$.

For the verification of (2.11) we need a third lemma.

Lemma 3 (Reduction of higher-order correlations; generalized Bayes' theorem)

For the set of higher-order correlation functions defined below we have (for nonvanishing denominators)

$$
\begin{gathered}
p_{\alpha \alpha^{\prime} ; \gamma}^{l ; l-1}=\left(p_{\alpha \gamma}^{(l-1)} / p_{\alpha \alpha^{\prime}}^{(l)}\right) p_{\alpha \gamma ; \alpha^{\prime}}^{(l-1 ; l)} \\
p_{\alpha \alpha^{\prime} ; \gamma_{1} \gamma_{2}}^{(l ; l-2, l-1)}=\left(p_{\alpha \gamma_{1}}^{(l-2)} p_{\alpha \gamma_{1} ; \gamma_{2}}^{(l-2 ;-1)} / p_{\alpha \alpha^{\prime}}^{(l)}\right) p_{\alpha \gamma_{1} \gamma_{2} ; \alpha^{\prime}}^{(l-2, l)}
\end{gathered}
$$

etc.

Proof. Consider the following higher-order correlations (multiple conditional probabilities) introduced via the joint probabilities on the left side

$$
\begin{gathered}
p\left(k=\alpha, k+l=\alpha^{\prime}\right)=p_{\alpha} p_{\alpha \alpha^{\prime}}^{(l)} \\
p\left(k=\alpha, k+l_{1}=\alpha_{1}, k+l_{2}=\alpha_{2}\right)=p_{\alpha} p_{\alpha \alpha_{1}}^{\left(l_{1}\right)} p_{\alpha \alpha_{1} ; \alpha_{2}}^{\left(l_{i} ; l_{2}\right)} \\
p\left(k=\alpha, k+l_{1}=\alpha_{1}, k+l_{2}=\alpha_{2}, k+l_{3}=\alpha_{3}\right) \\
=p_{\alpha} p_{\alpha \alpha_{1}}^{\left(l_{1}\right)} p_{\alpha \alpha_{1} \alpha_{2}}^{\left(l_{1} ; l_{2}\right)} p_{\alpha \alpha_{1} \alpha_{2} ; \alpha_{3}}^{\left(l_{1} l_{2} ; l_{3}\right)}
\end{gathered}
$$

etc. Here the symbol

$$
p_{\alpha_{0} \alpha_{1} \ldots \alpha_{n-1} ; \alpha}^{\left(l_{1} \ldots l_{n-1} ; l\right)}
$$

stands for the probability, subject to the set of constraints $f\left(k+l_{1}\right)=\alpha_{1}, \ldots, f\left(k+l_{n-1}\right)=\alpha_{n-1}, \quad$ for finding type $\alpha$ at a distance of $l$ domains from the (arbitrarily chosen) domain $k$ of type $\alpha_{0}$ ( $n$-point correlation).

There is also the following alternative representation:

$$
\begin{gathered}
p\left(k=\alpha, k+l=\alpha^{j}\right)=p_{\alpha} p_{\alpha \alpha^{\prime}}^{(l)} \\
p\left(k=\alpha, k+l_{1}=\alpha_{1}, k+l_{2}=\alpha_{2}\right)=p_{\alpha} p_{\alpha \alpha_{2}}^{\left(l_{2}\right)} p_{\alpha \alpha_{2} ; \alpha_{1}}^{\left(l_{2} l_{1}\right)} \\
p\left(k=\alpha, k+l_{1}=\alpha_{1}, k+l_{2}=\alpha_{2}, k+l_{3}=\alpha_{3}\right) \\
=p_{\alpha} p_{\alpha \alpha_{3}}^{\left(l_{3}\right)} p_{\alpha \alpha_{3} ; \alpha_{1} \alpha_{2}}^{\left(l_{3} ; l_{1} l_{2}\right)}
\end{gathered}
$$

etc. Here

$$
p_{\alpha_{0} \alpha_{n} ; \alpha_{1} \ldots \alpha_{n-1}}^{\left(l_{n} ; l_{1} \ldots l_{n-1}\right)}
$$

means the probability, subject to $f\left(k+l_{n}\right)=\alpha_{n}$, for finding the combination of types $\left(\alpha_{1}, \ldots, \alpha_{n-1}\right)$ at distances $\left(l_{1}, \ldots, l_{n-1}\right)$ from the (arbitrarily chosen) domain $k$ of type $\alpha_{0}$.

From both representations we can derive all higherorder correlation terms recursively. To this aim we use (2) and ( $\left.2^{\prime}\right)$ and set $l_{2}=l, l_{1}=l-1$. We get

$$
p_{\alpha} p_{\alpha \gamma}^{l-1} p_{\alpha \gamma ; \alpha^{\prime}}^{(l-1 ; l)}=p_{\alpha} p_{\alpha \alpha^{\prime}}^{l} p_{\alpha \alpha^{\prime} ; \gamma}^{(l, l-1)}
$$

and thus (A4.a).

Analogously it follows from (3) and (3'), setting $l_{3}=l, l_{2}=l-1, l_{1}=l-2$, that

$$
p_{\alpha} p_{\alpha \gamma_{1}}^{l-2} p_{\alpha \gamma_{1} ; \gamma_{2}}^{(l-2 ; l)} p_{\alpha \gamma_{1} \gamma_{2} ; \alpha^{\prime}}^{(l-2, l-1)}=p_{\alpha} p_{\alpha \alpha^{\prime}}^{l} p_{\alpha \alpha^{\prime} ; \gamma_{1} \gamma_{2}}^{(l ; l)-2,1-1)}
$$

and thus $(A 4 . b)$.

Iterating the procedure we finally get all conditional probabilities constrained to type combinations in terms of conditional probabilities constrained to one type only. We have thus shown the general recursive evaluability of all higher-order domain correlations in terms of the two-point functions. This finishes the proof by complete induction.

With Lemma 3 it is clear that only the one-type fixed probabilities

$$
P_{A}(n, l)=p_{\alpha_{1} \ldots \alpha_{n} ; \gamma}^{(l-n+1 \ldots l-1 ; l)} \quad \text { for all } n, l \in N
$$

are necessary for the calculation of all higher-order correlations in the term $\left.\left\langle F_{\mathrm{DB}}\right\rangle_{k}\right|_{\alpha \alpha^{\prime}}$ of (2.10).

The important observation is now that we can obtain the $P_{A}(n, l)$ directly from the transfer matrix $p_{\alpha \beta}$. In the most simple case - which we have assumed in (2.11) - of only next-neighbour domain interactions we get:

$$
\begin{array}{ll}
P_{A}(0, l)= & p_{\gamma} \\
P_{A}(1, l)=p_{\alpha_{1} ; \gamma}^{(l-1 ; l)}=p_{\alpha_{1} \gamma} \\
P_{A}(2, l)=p_{\alpha_{1} \alpha_{2} ; \gamma}^{(l-2, l ; 1 ; l)}=p_{\alpha_{2} \gamma} \\
P_{A}(3, l)=p_{\alpha_{1} \alpha_{2} \alpha_{3} ; \gamma}^{(l-3, l-2, l ; l)}=p_{\alpha_{3} \gamma}
\end{array}
$$

and so on. Thus

$$
p_{\alpha \alpha^{\prime} ; \gamma}^{(l ; l l-1)}=\left(p_{\alpha \gamma}^{(l-1)} / p_{\alpha \alpha^{\prime}}^{(l)}\right) p_{\gamma \alpha^{\prime}} .
$$

By expansion of the domain-distance term in terms of all possible length distributions of the domains between $k$ and $k+l$ we obtain

$$
\begin{aligned}
& \left.\left\langle\exp \left[i \mathbf{k}\left(\mathbf{r}_{k}-\mathbf{r}_{k+l}\right)\right]\right\rangle_{k}\right|_{\substack{\alpha, n_{k}=m \\
\alpha^{\prime}, n_{k+1}=n}} \\
= & \sum_{n^{\prime}} \sum_{\gamma} v_{\gamma^{\prime}}\left(n^{\prime}\right) \exp \left(-i \mathbf{k} \cdot n^{\prime} \mathbf{a}_{\gamma^{\prime}}\right)\left[p_{\alpha \alpha^{\prime} ; \gamma^{\prime}}^{(l ; l-1)}\right] \\
& \times\left.\left\langle\exp \left[i \mathbf{k} \cdot\left(\mathbf{r}_{k}-\mathbf{r}_{k+l-1}\right)\right]\right\rangle_{k}\right|_{\substack{\alpha, n_{k}=m \\
\gamma^{\prime}, n_{k+1-1}=n^{\prime}}}
\end{aligned}
$$

and combined with (A.6) we obtain the reduction formula (2.11) that we wanted to prove.

\section{References}

Adlhart, W. (1981). Acta Cryst. A37, 794-801.

Aspnes, D. E. \& IHM, J. (1986). Phys. Rev. Lett. 57, 3054-3057. Barthes-Labrousse, M.-G. (1986). Surf. Sci. 177, 338-352. BoulliaRD, J. C. \& SotTo, M. P. (1986). Surf. Sci. 177, 139-156. Cowley, J. M. (1976). Acta Cryst. A32, 83-91.

Cowley, J. M. \& AU, A. Y. (1978). Acta Cryst. A34, 738-743. 
Fenter, P. \& Lu, T.-M. (1985). Surf. Sci. 154, 15-21.

Hendricks, S. B. \& Teller, E. (1942). J. Chem. Phys. 10, 147-167.

Hosemann, R. \& BAGChI, S. N. (1962). Direct Analysis of Diffraction by Matter. Amsterdam: North Holland.

Houston, J. E. \& PARK, R. L. (1970). Surf. Sci. 21, 209-223.

Houston, J. E. \& PARK, R. L. (1971). Surf. Sci. 26, 269-285.

IOSIFESCU, M. (1980). Finite Markov Processes and their Applications. New York: Wiley.

IRANPOUR, R. \& CHACON, P. (1988). Basic Stochastic Processes. New York: Macmillan.

JAGODZINSKI, H. (1949). Acta Cryst. 2, 201-207, 208-214, 298304.

JAGODINSKI, H. (1987). Prog. Cryst. Growth Charact. Mater. 14, 47.

JAGODZINSKI, H. \& FREY, F. (1992). In International Tables for Crystallography, Vol. B, ch. 4.2, section 4.2.4.2. Dordrecht: Kluwer Academic Publishers. In the press.

JAGODZINSKI, H., MORITZ, W. \& WOLF, D. (1978). Surf. Sci. 77 , 233-248, 249-264, 265-282, 283-300.

Kakinoki, J. \& Komura, Y. (1952). J. Phys. Soc. Jpn, 7, 30-35. KaKinOKI, J. \& KomURA, Y. (1954). J. Phys. Soc. Jpn, 9, 169-183. KaKinOKi, J. \& Komura, Y. (1965). Acta Cryst. 19, 137-147.

Kern, K., Niehus, H., Schatz, A., ZepPenfeld, P., George, J. \& ComsA, G. (1991). Phys. Rev. Lett. 67, 855.
Landau, L. (1937). Phys. Z. Sowjetunion, 12, 579.

Lent, C. S. \& Cohen, P. I. (1984). Surf. Sci. 139, 121-154.

LifSCHITZ, I. M. (1937). Phys. Z. Sowjetunion, 12, 623.

Lu, T.-M. \& Lagally, M. G. (1982). Surf. Sci. 120, 47-66.

Neder, R. B., Frey, F. \& Schulz, H. (1990). Acta Cryst. A46, 792-798, 799-809.

Pimbley, J. M. \& Lu, T.-M. (1984). J. Appl. Phys. 55, 182-187.

Pimbley, J. M. \& Lu, T.-M. (1985a). J. Appl. Phys. 57, 1121-1129.

Pimbley, J. M. \& Lu, T.-M. (1985b). Surf. Sci. 159, 169-183.

Pimbley, J. M., Lu, T.-M. \& WANG, G.-C. (1985). Surf. Sci. 150, L467-L473.

Presicci, M. \& Lu, T.-M. (1984). Surf. Sci. 141, 233-239.

Pukite, P. R., Lent, C. S. \& Cohen, P. I. (1985). Surf. Sci. 161, 39-68.

Renaud, G., Fuoss, P. H., Bevk, J. \& Freer, B. S. (1992). Phys. Rev. B. Submitted.

Robinson, I. K., Vlieg, E., Hornis, H. \& Conrad, E. H. (1991). Phys. Rev. Lett. 67, 1890.

Seul, M. \& ToRney, D. C. (1989). Acta Cryst. A45, 381-396.

Welberry, T. R. (1985). Rep. Prog. Phys. 48, 1543-1593.

Wierenga, P. E., KubBy, J. A. \& Griffith, J. E. (1987). Phys. Rev. Lett. 59, 2169-2172.

Wilson, A. J. C. (1942). Proc. R. Soc. London Ser. A, 180, 277-285.

Wollschlaeger, J. \& Henzler, M. (1989). Phys. Rev. B, 39, 6052.

Acta Cryst. (1992). A48, 727-732

\section{Symbols for Symmetry Elements and Symmetry Operations \\ Final Report of the International Union of Crystallography Ad-Hoc Committee on the Nomenclature of Symmetry*}

By P. M. DE Wolff (Chairman), Meermanstraat 126, 2614 AM Delft, The Netherlands, Y. Billiet, Ecole Nationale d'Ingénieurs, BPW 3038 Sfax, Tunesië, J. D. H. DonnaY, Department of Geological Sciences, McGill University, 3450 University Street, Montreal, Canada H3A 2A7, W. FISCHER, Institut für Mineralogie, Petrologie und Kristallographie der Phillips-Universität, Lahnberge, D-3550 Marburg (Lahn), Germany, R. B. Galiulin, Institute of Crystallography, Academy of Sciences of Russia, Leninsky Prospekt 59, Moscow 117333, Russia, A. M. Glazer, Clarendon Laboratory, University of Oxford, Parks Road, Oxford OX13PU, England, TH. HAHN, Institut für Kristallographie, RWTH, Templergraben 55, D-5100 Aachen, Germany, MARJORIE Senechal, Department of Mathematics, Smith College, Northampton, MA 01063, USA, D. P. Shoemaker, Chemistry Department, Oregon State University, OR 97331, USA, H. WONDRATSCHEK, Institut für Kristallographie, Universität Karlsruhe, Kaiserstrasse 12, D-7500 Karlsruhe 1, Germany, A. J. C. WILson (ex officio, IUCr Commission on International Tables), Crystallographic Data Centre, University Chemistry Laboratory, Lensfield Road, Cambridge CB2 1EW, England and S. C. Abrahams (ex officio, IUCr Commission on Crystallographic Nomenclature), Department of Physics, Southern Oregon State College, 1250 Siskiyou Boulevard, Ashland, OR 97520-5047, USA

(Received 7 November 1991; accepted 9 March 1992)

\section{Abstract}

New or redefined printed symbols are proposed in the light of the recently accepted redefinition of sym-

\footnotetext{
* Appointed 14 November 1984, modified 10 August 1987 [see Acta Cryst. (1986), A42, 64 for original membership] under ground rules outlined in Acta Cryst. (1979), A35, 1072. Final Report accepted 23 December 1991 by the IUCr Commission on Crystallographic Nomenclature and 9 March 1992 by the Executive Committee.
}

metry elements [de Wolff et al. (1989). Acta Cryst. A45, 494-499]. In particular, the letter $e$ covers certain glide planes which hitherto had no unique symbol, such as those called 'either $a$ or $b$ '. The use of $e$ in the Hermann-Mauguin symbol of five different space groups is recommended. For $e$ planes projected in a direction parallel to the plane, a graphical symbol is proposed which removes the ambiguity of their present designation. The letter $k$ is proposed for a newly defined class of glide planes which until now were (C) 1992 International Union of Crystallography 